\title{
Habib, Samar (ed.). - Islam and Homosexuality | Kugle, Scott Siraj al-Haqq. - Homosexuality in Islam
}

Ludovic-Mohamed Zahed

\section{OpenEdition}

\section{Journals}

Édition électronique

URL : http://journals.openedition.org/etudesafricaines/14466

DOI : 10.4000/etudesafricaines. 14466

ISSN : 1777-5353

Éditeur

Éditions de l'EHESS

\section{Édition imprimée}

Date de publication : 28 mai 2013

Pagination : 468-471

ISBN : 978-2-7132-2387-7

ISSN : 0008-0055

Référence électronique

Ludovic-Mohamed Zahed, « Habib, Samar (ed.). - Islam and Homosexuality | Kugle, Scott Siraj al-Haqq. - Homosexuality in Islam », Cahiers d'études africaines [En ligne], 209-210 | 2013, mis en ligne le 27 mai 2013, consulté le 22 septembre 2020. URL : http://journals.openedition.org/etudesafricaines/14466 ; DOI : https://doi.org/10.4000/etudesafricaines.14466

Ce document a été généré automatiquement le 22 septembre 2020.

(c) Cahiers d'Études africaines 


\title{
Habib, Samar (ed.). - Islam and Homosexuality | Kugle, Scott Siraj al- Haqq. - Homosexuality in Islam
}

\author{
Ludovic-Mohamed Zahed
}

\section{RÉFÉRENCE}

HABIB, Samar (ed.). - Islam and Homosexuality. Vol. I. Westport, Praeger, 2009, 503 p., bibl.

KUGLE, Scott Siraj al-Haqq. - Homosexuality in Islam: Critical Reflection on Gay, Lesbian and Transgender Muslims. London, Oneworld Publications, 2010, 335 p., bibl.

Les études de genre appliquées à l'islam depuis la fin des années 1990, et plus particulièrement la place de ce que d'aucuns qualifient de « minorités sexuelles » dans le monde arabo-musulman contemporain, est l'objet de fascinantes et nouvelles orientations dans la recherche académique. Deux ouvrages, en 2009 et en 2010, tentent d'élaborer une représentation nouvelle des liens conceptuels et sociologiques établis désormais entre islam et homosexualité, par des approches radicalement différentes.

C'est ainsi que l'ouvrage de Samar Habib, Islam and Homosexuality, rassemble les points de vue de vingt experts explorant ces questions, afin de fournir un regard exhaustif sur les interactions entre individus de même sexe au sein des cultures arabo-musulmanes d'aujourd'hui. Samar Habib commence par nous livrer une longue introduction, afin de dresser une forme de constat à propos de ce que vivent les homosexuels au sein du monde arabe. L'auteure cite tout d'abord la première initiative publique entreprise par une association $\mathrm{LGBTQI}^{1}$ au Liban ; une première initiative qui contredisait à l'époque, en 2009, l'assertion occidentale selon laquelle les gays et lesbiennes n'existaient pas au sein d'une culture arabe qui, en l'occurrence, aurait été baignée dans une forme de bisexualité «envahissante $»^{2}$. Une assertion doublement problématique, selon Habib, puisqu'elle réduit les rapports sexuels entre individus de même sexe à un paradigme 
exclusivement occidental, tout en confondant comportement sexuel et identification à l'homosexualité en tant que telle.

Habib rappelle en cela l'importance de l'utilisation de l'acronyme LGBTQI qui repose, d'après elle, sur une conception " universelle » - platonicienne - des droits humains. Elle rappelle également que certains pays arabes nient tout bonnement le fait que les droits LGBTQI sont des droits humains ${ }^{3}$. L'auteure considère qu'une interprétation réactualisée des textes du Coran - Tafsir - a déjà commencé au sein du monde arabomusulman ; comme cela fut déjà tenté en Andalousie par des savants tels qu'Ibn Hazm Cette réforme de l'islam pourrait permettre aux pays arabes de revenir, à long terme, à une conception plus universelle des droits de l'être humain élaborée par les pays arabomusulmans. Habib précise également que, selon l'étude menée par Tilo Becker ${ }^{5}$, la culture de «l'homophobie» n'est pas exclusivement présente dans ces pays, au contraire. Par conséquent, Habib pense que ce n'est que lorsque les pays arabes parviendront à dépasser l'oppression colonialiste et postcolonialiste, au moment où ils parviendront à subvenir à leurs besoins les plus élémentaires - nourriture, logement, sécurité -, qu'ils seront à même de s'émanciper en développant des "valeurs émancipatrices » (vol. I, p. 57). Ainsi, l'islam étant élaboré comme toute religion au sein de la culture dont il est issu, ces valeurs axiologiques réformées, d'un monde arabe en pleine mutation, pourraient donner jour, selon l'auteure, à une représentation "gay friendly» de l'islam (vol. II, chap. XI) et des unions entre individus de même sexe au sein de l'espace sociétal arabe.

Habib conclut en affirmant que, selon elle, il serait « délétère » de considérer ceux et celles s'affirmant aujourd'hui au sein du monde arabo-musulman en tant que gays ou lesbiennes, comme appartenant tous à une élite « occidentalisée » (vol. I, introduction, section lvii). De la même manière, elle pense qu'une herméneutique islamique "queerfriendly $»^{6}$ ne sera possible tout d'abord que par le rejet des traditions prophétiques non authentiques - les hadiths -, sur lesquels les premiers savants musulmans ont pourtant élaboré leurs dogmes. Ensuite, il faudra " déprogrammer " (introduction, section LVIII) l'interprétation homophobe des versets du Coran qui parlent du peuple de Loth à Sodome et Gomorrhe. Enfin, la jurisprudence islamique se devra de reconnaitre l'union maritale des couples de même sexe, tout en continuant de considérer l'adultère et la fornication comme contraires aux valeurs de l'islam. L'objectif ultime de la « copulation », nous dit-elle, n'est pas la reproduction, mais bien « le principe de plaisir ainsi que l'harmonie sociale affirmée avec empathie » (ibid.).

L'ouvrage de Kugle, Homosexuality in Islam: Critical Reflection on Gay, Lesbian and Transgender Muslims, aux antipodes de l'approche sociologique développée par Habib et bien que visant le même objet, tente d'élaborer une «théologie libératrice » de toutes formes d'oppressions (p. 35) notamment en «embrassant un humanisme islamique» (p. 269). Son ouvrage est sans doute l'un des premiers à aborder la question de l'homosexualité de manière aussi engagée, tout en se focalisant sur l'examen méticuleux et «systématique " des textes religieux afin d'en proposer une représentation «alternative » (p.72). Dès le titre, l'auteur parle de l'homosexualité comme faisant, a priori, partie de l'islam, à la différence d'autres ouvrages - comme celui de Habib - qui ont un point de vue plus dichotomique sur la question, considérant en quelque sorte que les deux identités cohabitent d'une façon plus ou moins apaisée. Selon lui, les individus appartenant à ce que l'on considère aujourd'hui 
comme des «minorités sexuelles » auraient un point de vue privilégié sur ce qu'est l'islam.

Avant d'expliciter sa thèse, l'auteur postule que certains êtres humains sont homosexuels par "disposition» - facteurs de type génétique, hormonaux, sociaux plutôt que par choix (pp. 9, 253); il ajoute que si les textes religieux condamnent les rapports sexuels entre individus de même sexe, ce n'est que dans un contexte d'exploitation ou de violence (p.2). Le défi de cette thèse, comme tout travail de réforme progressiste, écrit-il (p. 3), est de parvenir à séparer ce qui relève de la culture " patriarcale ", de ce qui est essentiel à la foi, à la spiritualité. En cela, son paradigme se base essentiellement sur les témoignages d'activistes LGBT en Afrique du Sud, aux ÉtatsUnis, au Canada et aux Pays-Bas. Enfin, l'auteur précise que le paradigme utilisé souffre une limite : celle de postuler l'existence d'un dieu unique, dont le Coran serait la parole, qui aurait envoyé un messager sincère en la personne de Mahomet. L'objectif de cette thèse étant d'ouvrir un dialogue fait de ponts et d'interconnexions entre l'islam, en tant que tradition, et les musulmans en tant qu'êtres humains (p. 12).

L'auteur entame d'ailleurs son propos par un verset du Coran - fait original pour un professeur universitaire -, afin de rappeler que le Coran enjoint chacun de nous à prendre ses responsabilités; ce qu'il fit personnellement lors du procès du premier réfugié nord-américain, d'origine marocaine, qui disait fuir son pays « du fait qu'il avait été persécuté et abusé sexuellement en raison de son orientation sexuelle» (p. 16). Ce procès, qui s'est déroulé durant l'été 2002, posa pour la première fois aux États-Unis, de manière publique, la question de savoir « ce que dit l'islam de l'homosexualité » (p. 20). L'auteur rappelle que, bien qu'il ne puisse s'exprimer au nom de l'islam dans sa globalité, il s'est attelé à tenter d'apporter des éléments de réponse à cette question épineuse, dans un contexte géopolitique post-11-Septembre 2001, sans pour autant verser dans la caricature que serait un " procès de l'islam ». C'est dans cette perspective que Kugle nous rappelle combien les savants des premiers siècles de l'islam, comme son maître à penser en la matière $\mathrm{Ibn} \mathrm{Hazm}^{7}$, n'éprouvaient aucune pudeur à aborder tous les sujets sociétaux possibles, y compris celui de l'homosexualité. En cela, Ibn Hazm rappelait par exemple que l'amour est l'amour, qu'il soit hétérosexuel ou homosexuel ${ }^{8}$. L'influence des savants andalous s'est perdue au fil des âges et des influences politiques plus rigoristes.

Afin de mieux appréhender le substrat théologique sur lequel reposeraient de telles allégations de la part des fondamentalistes, Kugle consacre donc la plus grande part de son ouvrage à revivifier l'héritage de ces savants des premiers siècles de l'islam, par l'élaboration d'une démonstration selon laquelle islam et homosexualité ne seraient pas incompatibles. Il aborde bien entendu la question de l'exégèse coranique et des célèbres versets concernant les «sodomites » - que le Coran décrit comme le " peuple de Loth ». L'auteur conclut que les "gays » - ou même les individus transidentitaires ne sont jamais cités explicitement dans le Coran. Selon lui, il serait possible de leur faire une place au sein de la représentation de la Création portée par l'exégèse coranique (p. 72). Kugle poursuit sa démonstration par l'étude systématique, nous ditil, de tous les hadiths (traditions prophétiques) ayant trait directement ou indirectement à la question de l'homosexualité. Il conclut que, selon lui, là encore au vu des critères "classiques" élaborés par les savants du hadith, aucune tradition dite " authentique» ne condamnerait explicitement l'homosexualité en tant que telle. La démonstration de Kugle, contrairement à celle de Habib, s'inscrit dans la démarche 
d'un musulman ouvertement engagé pour une plus grande inclusivité au sein de l'islam contemporain.

Quoi qu'il en soit, l'approche révolutionnaire de ce type d'ouvrages engagés et originaux, est illustrée de manière très explicite par les propos de Parvez Sharma ${ }^{9}$ dans sa préface de l'ouvrage de Habib, qui insistent sur l'importance des études de genre appliquées au champ islamique: "Certains de nos combats les plus amères dans le siècle à venir auront lieu à l'avant-garde du champ religieux [...]. En effet, s'il doit y avoir un Jihad pour l'amour, alors ses martyrs devront partir de la croyance en la sainteté du Coran, et devront garder l'espoir qu'il est possible d'aller au-delà des limites de la théologie » (préface, p. 9).

\section{NOTES}

1. Lesbiennes, gays, bisexuel(le)s, transidentitaires, "queer », intersexuels. "Queer » est, à la base, un mot anglais signifiant "étrange ", " peu commun », souvent utilisé comme insulte envers des individus gays, lesbiennes, transsexuels. Bien que son sens argotique premier («louche», "pédé ») soit insultant, les homosexuels américains nomment ainsi la branche qui se voudrait être la plus activiste de leur mouvement, la plus politiquement contestataire, se voulant à l'avant-garde du conformisme dans lequel s'inscrirait un courant gay (homosexuel) assagi et démobilisé.

2. J. A. MASSAD(Desiring Arabs,Chicago, University of Chicago Press, 2007) critique notamment ce qu'il appelle « l'internationale gay » : une élite arabe qui chercherait à influencer la culture arabe afin de l'occidentaliser.

3. Habib cite la déclaration universelle islamique des droits humains (UIDHR) qui contient une clause selon laquelle les droits humains ne peuvent être définis en contradiction avec la «sharia islamique " (vol. I, introduction, section XxII), elle-même définie par le dogme imposé par des pays comme l'Arabie Saoudite ou l'Iran. Selon Habib, on trouve une excellente discussion à ce sujet dans l'ouvrage de E. BREMS,The Human Rights: Universality and Diversity, The Hague, Matinus Nijhoff Publishers, 2001.

4. Ce dernier considérant qu'il n'y avait aucun mal à ce qu'un jeune homme aime un autre jeune homme. Selon Ibn Hazm, le problème vient du fait que si l'acte sexuel est révélé au grand jour, la réputation des individus concernés n'est plus en accord avec la norme sociale attendue de la part de «l'élite "; c'est là d'ailleurs une forme de tabou encore en vigueur, sous cette forme véritablement, dans la plupart des pays arabo-musulmans (Ibn HAzM,The Ring of the Dove, London, Luzac \& Company, 1953).

5. Tilo Becker est l'auteur de l'un des chapitres du présent ouvrage ; voir également T. BECKER, «Sexual Morality: Existing Measurements in Cross-Cultural Research and Proposals for Linguistic and Methodological Improvements", Conference Paper, 2008, <http:// www.csdiworkshop.org/pdf/3mc2008_proceedings/session_79/Beckers.pdf>.

6. S. HABIB, « Queer-Friendly Islamic Hermeneutics ",ISIM Review, 21, 2008, pp. 30-31.

7. Théologien et juriste andalou du XI ${ }^{\mathrm{e}}$ siècle, qui tenta d'intégrer au débat théologique des éléments de philosophie et de logique. 
8. Ibn HAZM, The Ring of the Dove : A Treatise on the Art and Practice of Arab Love. Trans. A. J. Arberry. Luzac Oriental, 1997; disponible en ligne en anglais: <http://www.muslimphilosophy.com/ hazm/dove/index.html>.

9. Réalisateur du documentaire "Jihad for Love» dans lequel interviennent de nombreuxgaysou lesbiennes musulman(e)s, dont l'imam Muhsinh Hendricks d'Afrique du Sud, voir < $<$ ttp:// www.ajihadforlove.com/muhsin.html>. 